\title{
A Comparison of Gastrointestinal Toxicities between Intensity-Modulated Radiotherapy and Three-Dimensional Conformal Radiotherapy for Pancreatic Cancer
}

Kyong Joo Lee*, Hong In Yoon ${ }^{\dagger, \ddagger}$, Moon Jae Chung ${ }^{\S}$, Jeong Youp Park ${ }^{\S}$, Seungmin Bang ${ }^{\S}$, Seung-woo Park ${ }^{\S}$, Jin Sil Seong ${ }^{\dagger}$, and Si Young Song $\$$

"Division of Gastroenterology and Hepatology, Department of Internal Medicine, Yonsei University Wonju College of Medicine, Wonju, ${ }^{\dagger}$ Department of Radiation Oncology, ${ }^{\ddagger}$ Brain Korea 21 Plus Project for Medical Science, and ${ }^{s}$ Division of Gastroenterology, Department of Internal Medicine, Yonsei University College of Medicine, Seoul, Korea

See editorial on page 164.

Background/Aims: Concurrent chemoradiotherapy (CCRT) is considered the treatment option for locally advanced pancreatic cancer, but accompanying gastrointestinal toxicities are the most common complication. With the introduction of three-dimensional conformal radiotherapy (3-D CRT) and intensity-modulated radiotherapy (IMRT), CCRT-related adverse events are expected to diminish. Here, we evaluated the benefits of radiation modalities by comparing gastrointestinal toxicities between 3-D CRT and IMRT. Methods: Patients who received CCRT between July 2010 and June 2012 in Severance Hospital, Yonsei University College of Medicine, were enrolled prospectively. The patients underwent upper endoscopy before and 1 month after CCRT. Results: A total of 84 patients were enrolled during the study period. The radiotherapy modalities delivered included 3D-CRT $(n=40)$ and IMRT $(n=44)$. The median follow-up period from the start of CCRT was 10.6 months (range, 3.8 to 29.9 months). The symptoms of dyspepsia, nausea/vomiting, and diarrhea did not differ between the groups. Upper endoscopy revealed significantly more gastroduodenal ulcers in the 3-D CRT group $(p=0.003)$. The modality of radiotherapy (3D-CRT; odds ratio [OR], 11.67; $p=0.011$ ) and tumor location (body of pancreas; OR, 11.06; $p=0.009$ ) were risk factors for gastrointestinal toxicities. Conclusions: IMRT is associated with significantly fewer gastroduodenal injuries among patients treated with CCRT for pancreatic cancer. (Gut Liver 2016;10:303-309)
Key Words: Gastrointestinal toxicities; Chemoradiotherapy; Pancreatic neoplasms; Endoscopy; Radiotherapy, intensitymodulated

\section{INTRODUCTION}

Concurrent chemoradiotherapy (CCRT) can be a treatment option for locally advanced pancreatic cancer (LAPC). ${ }^{1,2}$ Since complete surgical resection is the only curative treatment, a vast majority of research has been targeted for treatments that could change the status of nonoperable pancreatic cancer to that of operable. Although some issues remain under debate, preoperative CCRT is currently being extended to resectable and borderline resectable pancreatic cancer. ${ }^{3-6}$ This treatment improves local control and resectability, and increases the possibility of R0 resection. ${ }^{7,8}$ However, as the stomach and small bowel are located close to the pancreas, both organs are included in the radiation field. CCRT-related gastrointestinal (GI) toxicities are major dose-limiting factors. ${ }^{9-11}$ Acute toxicities include nausea, vomiting, diarrhea, abdominal pain, and bleeding. Late toxicities related with radiotherapy are obstruction, stricture, and fistula formation. ${ }^{10,12}$ These toxicities tend to lower the patient's quality of life and can even delay treatment of pancreatic cancer, thus decreasing the chance of surgery.

With technological improvements in radiotherapy, the delivery modality has evolved from conventional to three-dimensional conformal radiotherapy (3-D CRT). Recently developed intensity-modulated radiotherapy (IMRT) allows a combination of variable radiation intensity and 3-D radiation treatment planning. IMRT is therefore superior to 3-D CRT in maximiz-

\footnotetext{
Correspondence to: Si Young Song

Division of Gastroenterology, Department of Internal Medicine, Yonsei University College of Medicine, 50-1 Yonsei-ro, Seodaemun-gu, Seoul 120752, Korea

Tel: +82-2-2228-1957, Fax: +82-2-393-6884, E-mail: sysong@yuhs.ac

Received on April 20, 2015. Revised on June 1, 2015. Accepted on June 15, 2015. Published online October 19, 2015 pISSN 1976-2283 eISSN 2005-1212 http://dx.doi.org/10.5009/gnl15186

@ This is an Open Access article distributed under the terms of the Creative Commons Attribution Non-Commercial License (http://creativecommons.org/licenses/by-nc/4.0) which permits unrestricted non-commercial use, distribution, and reproduction in any medium, provided the original work is properly cited.
} 
ing homogeneous radiation to the planned target volume, while minimizing the damage to adjacent normal tissues. ${ }^{13,14}$ Helical tomotherapy (HT) is a type of IMRT that allows for adjustment of the planned target volume using real-time treatment monitoring by diagnostic computed tomography (CT) systems. ${ }^{15}$ However, there have been no randomized trials comparing IMRT and 3-D CRT in terms of GI toxicities and other outcomes. In this study, we compared the outcomes of IMRT and 3-D CRT, using GI toxicities, including endoscopic findings, as the end point.

\section{MATERIALS AND METHODS}

\section{Patients}

The patients who received CCRT for treatment of borderline resectable pancreatic cancer and LAPC at Severance Hospital in Seoul, Korea, between July 2010 and June 2012 were prospectively enrolled. The enrollment criteria were pathologicallyproven pancreatic adenocarcinoma, age over 20 years and an Eastern Cooperative Oncology Group performance status of 0 to 1. The excluded patients were who had received chemotherapy or surgery before CCRT and advanced pancreatic cancer. The patients who were taking proton pump inhibitor were excluded but GI medication such as antiemetic and prokinetic drugs were allowed. The patients who had not completed their scheduled radiation therapy were also excluded from per-protocol analysis. All patients participating in this study provided informed consent. This trial was approved by the Institutional Review Board for Human Research at Severance Hospital (4-2011-0594).

\section{Staging}

All patients underwent dynamic CT scans and tumors were classified as resectable, locally advanced or advanced pancreatic cancer using the American Joint Committee on Cancer (seventh edition) TNM staging system. Borderline resectable pancreatic cancer was defined as tumor abutment of the superior mesenteric artery involving less than 180 degrees of the circumference of the artery, severe unilateral superior mesenteric vein and portal vein impingement and gastroduodenal artery encasement up to origin at the hepatic artery. ${ }^{16}$

\section{Chemotherapy}

The regimens of chemotherapy were gemcitabine- or 5-fluorouracil (5-FU)-based: (1) $1,000 \mathrm{mg} / \mathrm{m}^{2}$ of gemcitabine on days 1,8 , and 15 of a 4 -week regimen; (2) $1,000 \mathrm{mg} / \mathrm{m}^{2}$ of 5 -FU on days 1 to 3 of a 4-week regimen intravenously, or $0.5 \mathrm{~g}$ tegafur-uracil (UFT) three times per day orally until radiotherapy ended. Theses chemotherapy regimens were decided by Korean insurance standards. The response of treatment was evaluated 1 month after the end of chemoradiotherapy, according to the Response Evaluation Criteria in Solid Tumors. We continued combination therapy of gemcitabine and cisplatin for those who were unable to undergo surgery. The chemotherapy schedule was as following: $1,000 \mathrm{mg} / \mathrm{m}^{2}$ of gemcitabine on days 1,8 , and 15 and $70 \mathrm{mg} / \mathrm{m}^{2}$ of cisplatin on day 1 of a 4-week regimen intravenously.

\section{Radiotherapy}

The radiotherapy modalities of CCRT were either 3-D CRT using a 10-MV linear accelerator or IMRT using HT (Accuray, Sunnyvale, CA, USA). For IMRT, the patients received a respiration education to reduce intrafractional respiratory motion and to increase patient's set up accuracy. Additionally, the BodyFIX immobilization device (Medical Intelligence, Schwabmunchen, Germany) with a total body cover sheet and a full-body vacuum-locking bag was applied. When four-dimensional CT simulation was performed, intravenous contrast material and diluted oral gastrografin were applied precisely to delineate organs at risk in GI tract. We defined the gross tumor volume (GTV) as the primary tumor and involved regional lymph nodes. We defined the planning target volume (PTV) as the GTV plus a 5-mm margin. We applied a smaller margin if the adjacent duodenum was abutting GTV. For planning of IMRT, we used HT Hi-Art System version 2.0 (TomoTherapy Inc., Madison, WI, USA). ${ }^{17}$ At least $95 \%$ of the GTV must be covered by the prescription dose, and the maximal dose to contiguous $2 \mathrm{~mL}$ of duodenum was limited to 50 Gy (equivalent dose in 2 Gy), similar to 3-D CRT cases. The median fraction and total doses were 2.54 Gy (range, 2.5 to 2.85 Gy) and 58.42 Gy (range, 50 to 62.5 Gy), respectively. For 3-D CRT, GTV and PTV were also defined such as IMRT. The field arrangement was a three-field technique, which consisted of opposed laterals and an anteroposterior field or a four-field box technique. A total dose of 45 or 50.4 Gy was delivered in daily fractions of $1.8 \mathrm{~Gy} .{ }^{18}$ 3-D CRT and IMRT were performed 5 days per week. The patient decided the radiotherapy modality after detailed explanation by a radiology oncologist. CCRT was proceeded on weekdays.

\section{GI toxicities}

All patients underwent upper endoscopy before and 1 month after CCRT. The upper endoscopy was conducted by a boardcertified endoscopist and the result was verified by another board-certified endoscopist. Any patients with gastroduodenal ulcers at initial endoscopic findings were excluded. The patients were asked about any CCRT-related symptoms every week during radiotherapy in the outpatient department. After CCRT ended, patients were followed up once per month. CCRT-related GI symptoms included dyspepsia, nausea, vomiting, and diarrhea. GI toxicities were classified according to the National Cancer Institute Common Terminology Criteria for Adverse Events version 4.0. In our study, radiation-induced injuries observed with endoscopy were defined as telangiectasia, diffuse erythema of mucosa, ulcers, and scar formation. ${ }^{19}$ All patients with symptoms or signs of ulceration in endoscopic findings received 
proper medical treatments.

\section{Statistical analysis}

The Pearson chi-square test was used to compare the treatment results and GI toxicity between 3D-CRT and IMRT. The Kaplan-Meier method and log-rank tests were used to compare overall survival (OS) between 3-D CRT and IMRT. To determine risk factors of GI toxicity, we used logistic regression for multivariate analysis.

Table 1. Baseline Characteristics

\begin{tabular}{lccc}
\hline \multicolumn{1}{c}{ Characteristic } & 3-D CRT (n=40) & IMRT (n=44) & p-value \\
\hline Age, yr & $64.3 \pm 10.3$ & $61.5 \pm 9.5$ & 0.137 \\
Sex & & & 0.932 \\
$\quad$ Male & $24(60)$ & $26(59.1)$ & \\
$\quad$ Female & $16(40)$ & $18(40.9)$ & \\
Hypertension & $17(42.5)$ & $15(46.9)$ & 0.428 \\
Diabetes mellitus & $12(30)$ & $12(27.3)$ & 0.782 \\
Stage & & & 0.122 \\
$\quad$ Borderline resectable & $10(25)$ & $18(40.9)$ & \\
$\quad$ Locally advanced & $30(75)$ & $26(59.1)$ & \\
Tumor location & & & 0.517 \\
$\quad$ Head & $24(60)$ & $29(65.9)$ & \\
$\quad$ Body & $13(32.5)$ & $14(31.8)$ & \\
$\quad$ Tail & $3(7.5)$ & $1(2.3)$ & \\
Chemotherapy regimen & & & 0.001 \\
$\quad$ Gemcitabine & $18(45)$ & $39(88.6)$ & \\
$\quad$ 5-FU & $22(55)$ & $5(11.4)$ & \\
Tumor size, cm & $3.6 \pm 1.1$ & $3.4 \pm 1.1$ & 0.408 \\
CA 19-9, U/mL & $1,032.7 \pm 2,194.4$ & $1,327.8 \pm 2,145.7$ & 0.535 \\
CEA, ng/mL & $5.7 \pm 9.5$ & $8.6 \pm 15.6$ & 0.314 \\
Hemoglobin, g/dL & $13.1 \pm 1.6$ & $13.4 \pm 1.3$ & 0.550 \\
\hline
\end{tabular}

Data are presented as mean \pm SD or number $(\%)$.

3-D CRT, three-dimensional conformal radiotherapy; IMRT, intensitymodulated radiotherapy; 5-FU, 5-fluorouracil; CA 19-9, carbohydrate antigen 19-9; CEA, carcinoembryonic antigen.

Table 2. Comparison of Treatment Results according to Radiation Modality

\begin{tabular}{lccc}
\hline & 3-D CRT $(\mathrm{n}=40)$ & IMRT $(\mathrm{n}=44)$ & $\mathrm{p}$-value \\
\hline Treatment response & & & 0.216 \\
$\quad$ PR-SD & $25(62.5)$ & $33(75.0)$ & \\
$\quad$ PD & $15(37.5)$ & $11(25.0)$ & \\
Treatment after CCRT & & & 0.095 \\
$\quad$ Surgery & $3(9.1)$ & $10(23.8)$ & \\
$\quad$ Chemotherapy & $30(90.9)$ & $32(76.2)$ & \\
\hline
\end{tabular}

Data are presented as number (\%).

3-D CRT, three-dimensional conformal radiotherapy; IMRT, intensitymodulated radiotherapy; PR-SD, partial response-stable disease; PD, progressive disease; CCRT, concurrent chemoradiotherapy.
All analyses were performed using statistical software SPSS version 17 (SPSS Inc., Chicago, IL, USA). p-values less than 0.05 indicated statistical significance.

\section{RESULTS}

\section{Patient characteristics}

A total of 84 patients were enrolled in this study. Forty patients underwent 3-D CRT and 44 patients received IMRT. The median follow-up was 10.6 months (range, 3.8 to 29.9 months). The 3-D CRT and IMRT groups showed similar baseline characteristics, with the exception of chemotherapy regimen (Table 1). The IMRT group was more likely to have received gemcitabine than the 3-D CRT group ( $<<0.001)$.

\section{Comparison of treatment results and OS}

The treatment response was evaluated 1 month after the end of CCRT and the operability was also re-evaluated at the time (Table 2). Thirteen patients were downstaged and underwent surgery. R0 resection was performed in all patients. In the IMRT group, 10 patients (23.8\%) were downstaged and in 3-D CRT group, three patients $(9.1 \%)$ were downstaged and underwent surgery, but the difference was not significant $(p=0.095)$. The IMRT group showed significantly better OS than the 3-D CRT group, with a mean OS of 22.6 months compared with 15.8 months, and 1-year OS of $84 \%$ compared with $58 \%$, respectively ( $\mathrm{p}=0.006$ ) (Fig. 1).

\section{GI toxicities}

The overall incidence of GI toxicities, including dyspepsia, nausea/vomiting, and diarrhea, was similar between the two groups (Table 3). There was no incidence of grade 3 to 4 toxicity. Out of a total of 84 patients, 59 patients (70.2\%) underwent follow-up upper endoscopy 1 month after the end of CCRT. There were no patients with gastric or duodenal ulcers at the

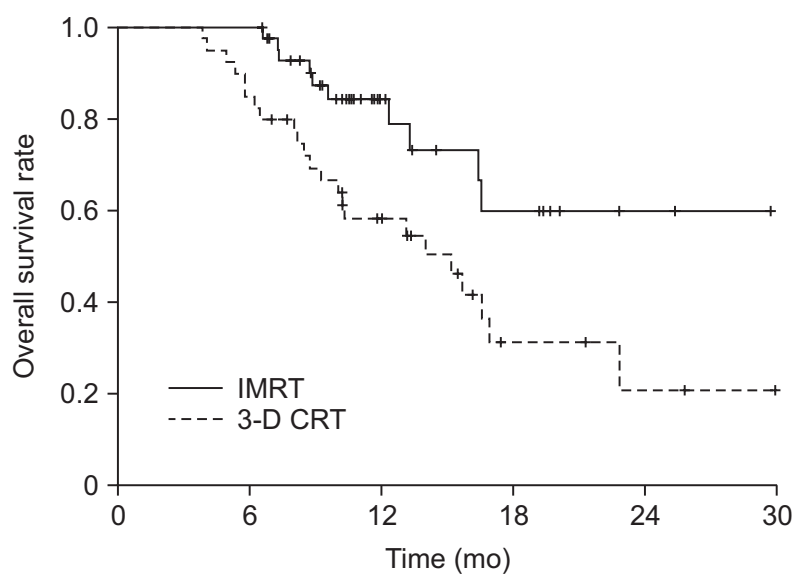

Fig. 1. Comparison of overall survival according to radiation modality. IMRT, intensity-modulated radiotherapy; 3-D CRT, three-dimensional conformal radiotherapy. 
initial endoscopic examination. However, in the follow-up endoscopy, the IMRT group showed a significantly lower incidence of gastroduodenal ulceration compared to the 3-D CRT group

Table 3. Comparison of Gastrointestinal Toxicity according to Radiation Modality

\begin{tabular}{lccc}
\hline & 3-D CRT (n=40) & IMRT (n=44) & $\mathrm{p}$-value \\
\hline $\begin{array}{l}\text { Dyspepsia } \\
\text { Grade 0 }\end{array}$ & $31(77.5)$ & $37(84.1)$ & 0.442 \\
$\quad$ Grade 1-2 & $9(22.5)$ & $7(15.9)$ & \\
Nausea/vomiting & & & 0.317 \\
$\quad$ Grade 0 & $26(65.0)$ & $33(75.0)$ & \\
$\quad$ Grade 1-2 & $14(35.0)$ & $11(25.0)$ & \\
Diarrhea & & & 0.725 \\
$\quad$ Grade 0 & $38(95.0)$ & $41(93.2)$ & \\
$\quad$ Grade 1-2 & $2(5.0)$ & $3(6.8)$ & \\
Upper endoscopy after CCRT & $26(65.0)$ & $33(75.0)$ & 0.317 \\
Gastroduodenal ulcers* & & & 0.003 \\
$\quad$ Grade 0 & $15(57.7)$ & $30(90.9)$ & \\
$\quad$ Grade 1-2 & $11(42.3)$ & $3(9.1)$ & \\
\hline
\end{tabular}

Data are presented as number $(\%)$.

3-D CRT, three-dimensional conformal radiotherapy; IMRT, intensitymodulated radiotherapy; CCRT, concurrent chemoradiotherapy.

*Gastroduodenal ulcers were evaluated by endoscopy.
( $\mathrm{p}=0.003)$. GI bleeding or perforation was not observed during the follow-up period in either group. The tumor location (body of pancreas; odds ratio [OR], 11.06; $p=0.009$ ) and 3-D CRT (OR, $11.67 ; \mathrm{p}=0.011$ ) were independent risk factors of GI toxicity (Table 4).

\section{DISCUSSION}

CCRT was first attempted for LAPC by the Gastrointestinal Tumor Study Group (GITSG) in the 1980 s. $^{20}$ Since then, many trials have been carried out and have showed promising results, but the use of CCRT in pancreatic cancer is still under debate. A few large randomized studies were carried out after the GITSG study. However, the radiation dose and chemotherapy regimens differed. The E4201 study demonstrated a benefit of chemoradiotherapy over chemotherapy using 3-D CRT and gemcitabine. Recently, CCRT is being carried out in resectable and borderline resectable pancreatic cancer as a neoadjuvant treatment. ${ }^{21,22}$ The rationale for preoperative CCRT includes downstaging of tumors, prevention of micrometastasis and selection of those patients with early far metastasis during treatment. ${ }^{23,24}$ Preoperative CCRT has thus led to improvement of resectability, Ro resection and prevention of recurrence after curative surgery. In addition, unnecessary surgery can be avoided for those patients with early far metastatic pancreatic cancer.

Table 4. Univariate and Multivariate Analyses for Risk Factors of Gastrointestinal Toxicity

\begin{tabular}{|c|c|c|c|c|c|}
\hline Variable & GI toxicity & p-value* & OR & $95 \% \mathrm{CI}$ & $p$-value \\
\hline Age, yr & & 0.564 & & & \\
\hline$\leq 65$ & $9(64.3)$ & & 1 & & \\
\hline$>65$ & $5(35.7)$ & & 0.39 & $0.07-2.03$ & 0.265 \\
\hline Sex & & 0.200 & & & \\
\hline Male & $6(42.9)$ & & 1 & & \\
\hline Female & $8(57.1)$ & & 0.36 & $0.07-1.77$ & 0.209 \\
\hline Tumor location & & 0.024 & & & \\
\hline Head of pancreas & $6(42.9)$ & & 1 & & \\
\hline Body & $8(57.1)$ & & 11.06 & $1.84-66.51$ & 0.009 \\
\hline Tail & 0 & & & & \\
\hline Radiation modality & & 0.003 & & & \\
\hline IMRT & $3(21.4)$ & & 1 & & \\
\hline 3-D CRT & $11(78.6)$ & & 11.67 & $1.74-78.1$ & 0.011 \\
\hline Chemotherapy regimen & & 0.184 & & & \\
\hline Gemcitabine & $8(57.1)$ & & 1 & & \\
\hline 5-FU & $6(42.9)$ & & 1.14 & $0.20-6.41$ & 0.881 \\
\hline Tumor size, $\mathrm{cm}$ & & 0.406 & & & \\
\hline$\leq 3.5$ & $8(57.1)$ & & 1 & & \\
\hline$>3.5$ & $6(42.9)$ & & 0.67 & $0.13-3.45$ & 0.634 \\
\hline
\end{tabular}

Data are presented as number (\%).

GI, gastrointestinal; OR, odds ratio; CI, confidence interval; IMRT, intensity-modulated radiotherapy; 3-D CRT, three-dimensional conformal radiotherapy; 5-FU, 5-fluorouracil.

${ }^{*}$ Chi-square test was used; ${ }^{\dagger}$ Logistic regression was used. 
The biggest concern associated with CCRT is the complication of nearby structures, especially the small bowel and stomach. As the pancreas is moderately sensitive to radiotherapy, higher doses of radiation are needed and complications are inevitable. ${ }^{25}$ Conventional radiotherapy contributed to the high incidence of GI toxicity before the early 1990 s. $^{26}$ However, with advancements in radiotherapy, 3-D CRT could reduce GI toxicities by using CT images for guidance. IMRT is a more recent modality to deliver radiotherapy. IMRT can provide higher and homogeneous radiation doses to the target planned volume and minimize toxicities to the adjacent critical structures. ${ }^{7}$ Yovino et al. ${ }^{12}$ showed that the overall incidence of grade 3 to 4 acute GI toxicity was low in patients who received IMRT-based CCRT compared to 3-D CRT. However, in our study, there was no grade 3 to 4 GI toxicity in either the IMRT or 3-D CRT groups. When comparing grade 1 to 2 GI toxicity between treatment modalities, the symptoms of dyspepsia, nausea/vomiting, and diarrhea were not significantly different because most of the patients received medications, such as antiemetics, during treatment. However, the incidence of grade 1 to 2 gastroduodenal ulceration was significantly higher in the 3-D CRT group than in the IMRT group. The endoscopic findings showed that IMRT could minimize the radiation to organs at risk. Nakamura et al. ${ }^{10}$ showed that the estimated incidence of upper GI bleeding at 1 year was about 20\% in those patients who received 3-D CRT with weekly gemcitabine. In addition, our previous study showed similar GI bleeding rate and 24.5\% of GI bleedings occurred less than 3 months after CCRT. ${ }^{9}$ Based on our previous data, we performed upper endoscopy at 1 month after the end of CCRT to evaluate the early events. In this study, there was no incidence of GI bleeding during the follow-up period, because the patients who showed abnormal endoscopic findings received proper medical management, such as a proton pump inhibitor. Late toxicities such as obstruction, perforation and fistula, were not found in this study.

Until recently, there have been few studies which compare the outcomes and OS between IMRT group and 3-D CRT group. Combs et al. $^{27}$ reported the OS and progression-free survival were not different in IMRT group and 3-D CRT group. However, in our study, the IMRT group showed improved OS compared to the 3-D CRT group. Although the objective response and the rate of surgery after CCRT were not significantly different, more patients who received IMRT seemed to undergo surgery $(\mathrm{p}=0.095)$ by downstaging the tumor. IMRT allows for delivery of higher doses and homogeneous radiation to the planned target volume and adjustment of the radiation field in real-time. ${ }^{28}$ One previous study that was conducted in our institute demonstrated that high-dose HT with concurrent full-dose chemotherapy resulted in improved local control and long-term survival in patients with LAPC. $^{29}$ The toxicities were acceptable. Also, full dose of gemcitabine was used in this study compared to $300 \mathrm{mg} / \mathrm{m}^{2}$ of gemcitabine used in Combs study. In our previous studies, full-dose gemcitabine based CCRT were treated in LAPC and the toxicities were acceptable. ${ }^{29-31}$ These advantages may delay tumor progression by local control and prevent micrometastasis of the tumor and lead to better resectability.

We note that our study had some limitations. Firstly, the chemotherapy regimen was significantly different between the two groups. Most of the patients in the IMRT group received gemcitabine. There are several reports that gemcitabine-based chemoradiotherapy is associated with better OS than 5-FUbased chemoradiotherapy. ${ }^{32,33}$ Also, in this study, the OS was higher in patients with gemcitabine than those with 5-FU/ UFT (20.6 months vs 9.4 months, $\mathrm{p}<0.001)$. In Korean medical policy, patients receive insurance benefits for 3-D CRT but not for IMRT. Thus, administration of chemotherapy depends on the radiation modality. Therefore, in the 3-D CRT group, the chemotherapy regimen was selected based on patients' economic status. Although this study was a prospective cohort trial, the chemotherapy regimen could not be unified. Second, the stages were not significantly different in both groups, but more LAPC patients seemed to be included in the 3-D CRT group. Third, we could not compare the dose-volume parameters between two modalities, so, in further study, comparison of dose volume parameters according to radiation modalities and the correlation between dose volume parameters and GI toxicities should be evaluated.

In conclusion, IMRT-based chemoradiotherapy significantly reduced GI toxicities which act as limiting factors for the treatment. In addition, IMRT improved the treatment results of pancreatic cancer. Large and well-designed randomized trials are needed to decide on a standard radiation modality and chemotherapy regimen.

\section{CONFLICTS OF INTEREST}

No potential conflict of interest relevant to this article was reported.

\section{REFERENCES}

1. Huguet F, Girard N, Guerche CS, Hennequin C, Mornex F, Azria D. Chemoradiotherapy in the management of locally advanced pancreatic carcinoma: a qualitative systematic review. J Clin Oncol 2009;27:2269-2277.

2. Loehrer PJ Sr, Feng Y, Cardenes H, et al. Gemcitabine alone versus gemcitabine plus radiotherapy in patients with locally advanced pancreatic cancer: an Eastern Cooperative Oncology Group trial. J Clin Oncol 2011;29:4105-4112.

3. Evans DB, Varadhachary GR, Crane CH, et al. Preoperative gemcitabine-based chemoradiation for patients with resectable adenocarcinoma of the pancreatic head. J Clin Oncol 2008;26:3496-3502. 
4. Le Scodan R, Mornex F, Girard N, et al. Preoperative chemoradiation in potentially resectable pancreatic adenocarcinoma: feasibility, treatment effect evaluation and prognostic factors, analysis of the SFRO-FFCD 9704 trial and literature review. Ann Oncol 2009;20:1387-1396.

5. Brunner TB, Scott-Brown M. The role of radiotherapy in multimodal treatment of pancreatic carcinoma. Radiat Oncol 2010;5:64.

6. Palta M, Willett C, Czito B. Role of radiation therapy in patients with resectable pancreatic cancer. Oncology (Williston Park) 2011; 25:715-721, 727.

7. Wilkowski R, Thoma M, Weingandt H, Dühmke E, Heinemann V. Chemoradiation for ductal pancreatic carcinoma: principles of combining chemotherapy with radiation, definition of target volume and radiation dose. JOP 2005;6:216-230.

8. Varadhachary GR. Preoperative therapies for resectable and borderline resectable pancreatic cancer. J Gastrointest Oncol 2011;2:136142.

9. Lee KJ, Kim HM, Jung JW, et al. Gastrointestinal hemorrhage after concurrent chemoradiotherapy in locally advanced pancreatic cancer. Gut Liver 2013;7:106-111.

10. Nakamura A, Shibuya K, Matsuo Y, et al. Analysis of dosimetric parameters associated with acute gastrointestinal toxicity and upper gastrointestinal bleeding in locally advanced pancreatic cancer patients treated with gemcitabine-based concurrent chemoradiotherapy. Int J Radiat Oncol Biol Phys 2012;84:369-375.

11. Kavanagh BD, Pan CC, Dawson LA, et al. Radiation dose-volume effects in the stomach and small bowel. Int J Radiat Oncol Biol Phys 2010;76(3 Suppl):S101-S107.

12. Yovino S, Poppe M, Jabbour S, et al. Intensity-modulated radiation therapy significantly improves acute gastrointestinal toxicity in pancreatic and ampullary cancers. Int J Radiat Oncol Biol Phys 2011;79:158-162.

13. Kataria T, Rawat S, Sinha SN, et al. Intensity modulated radiotherapy in abdominal malignancies: our experience in reducing the dose to normal structures as compared to the gross tumor. J Cancer Res Ther 2006;2:161-165.

14. Taylor R, Opfermann K, Jones BD, et al. Comparison of radiation treatment delivery for pancreatic cancer: linac intensity-modulated radiotherapy versus helical tomotherapy. J Med Imaging Radiat Oncol 2012;56:332-337.

15. Mackie TR, Holmes T, Swerdloff S, et al. Tomotherapy: a new concept for the delivery of dynamic conformal radiotherapy. Med Phys 1993;20:1709-1719.

16. Callery MP, Chang KJ, Fishman EK, Talamonti MS, William Traverso L, Linehan DC. Pretreatment assessment of resectable and borderline resectable pancreatic cancer: expert consensus statement. Ann Surg Oncol 2009;16:1727-1733.

17. Combs SE, Konkel S, Schulz-Ertner D, et al. Intensity modulated radiotherapy (IMRT) in patients with carcinomas of the paranasal sinuses: clinical benefit for complex shaped target volumes. Radiat Oncol 2006;1:23.
18. Koom WS, Seong J, Kim YB, Pyun HO, Song SY. CA 19-9 as a predictor for response and survival in advanced pancreatic cancer patients treated with chemoradiotherapy. Int J Radiat Oncol Biol Phys 2009;73:1148-1154.

19. DeCosse JJ, Rhodes RS, Wentz WB, Reagan JW, Dworken HJ, Holden WD. The natural history and management of radiation induced injury of the gastrointestinal tract. Ann Surg 1969;170:369384.

20. Moertel CG, Frytak S, Hahn RG, et al. Therapy of locally unresectable pancreatic carcinoma: a randomized comparison of high dose (6000 rads) radiation alone, moderate dose radiation (4000 rads + 5-fluorouracil), and high dose radiation + 5-fluorouracil: The Gastrointestinal Tumor Study Group. Cancer 1981;48:1705-1710.

21. Springett GM, Hoffe SE. Borderline resectable pancreatic cancer: on the edge of survival. Cancer Control 2008;15:295-307.

22. Belli C, Cereda S, Anand S, Reni M. Neoadjuvant therapy in resectable pancreatic cancer: a critical review. Cancer Treat Rev 2013;39:518-524.

23. Strobel O, Berens V, Hinz U, et al. Resection after neoadjuvant therapy for locally advanced, "unresectable" pancreatic cancer. Surgery 2012;152(3 Suppl 1):S33-S42.

24. Papalezova KT, Tyler DS, Blazer DG 3rd, et al. Does preoperative therapy optimize outcomes in patients with resectable pancreatic cancer? J Surg Oncol 2012;106:111-118.

25. Dobelbower RR Jr. The radiotherapy of pancreatic cancer. Semin Oncol 1979;6:378-389.

26. Wang F, Kumar P. The role of radiotherapy in management of pancreatic cancer. J Gastrointest Oncol 2011;2:157-167.

27. Combs SE, Habermehl D, Kessel K, et al. Intensity modulated radiotherapy as neoadjuvant chemoradiation for the treatment of patients with locally advanced pancreatic cancer: outcome analysis and comparison with a 3D-treated patient cohort. Strahlenther Onkol 2013;189:738-744.

28. Poppe MM, Narra V, Yue NJ, Zhou J, Nelson C, Jabbour SK. A comparison of helical intensity-modulated radiotherapy, intensitymodulated radiotherapy, and 3D-conformal radiation therapy for pancreatic cancer. Med Dosim 2011;36:351-357.

29. Chang JS, Wang ML, Koom WS, et al. High-dose helical tomotherapy with concurrent full-dose chemotherapy for locally advanced pancreatic cancer. Int J Radiat Oncol Biol Phys 2012;83:14481454.

30. Chung HW, Bang SM, Park SW, et al. A prospective randomized study of gemcitabine with doxifluridine versus paclitaxel with doxifluridine in concurrent chemoradiotherapy for locally advanced pancreatic cancer. Int J Radiat Oncol Biol Phys 2004;60: 1494-1501.

31. Cho IR, Chung MJ, Bang S, et al. Gemcitabine based neoadjuvant chemoradiotherapy therapy in patients with borderline resectable pancreatic cancer. Pancreatology 2013;13:539-543.

32. Zhu CP, Shi J, Chen YX, Xie WF, Lin Y. Gemcitabine in the chemoradiotherapy for locally advanced pancreatic cancer: a 
meta-analysis. Radiother Oncol 2011;99:108-113.

33. Huang J, Robertson JM, Margolis J, et al. Long-term results of full-dose gemcitabine with radiation therapy compared to 5-fluo- rouracil with radiation therapy for locally advanced pancreas cancer. Radiother Oncol 2011;99:114-119. 